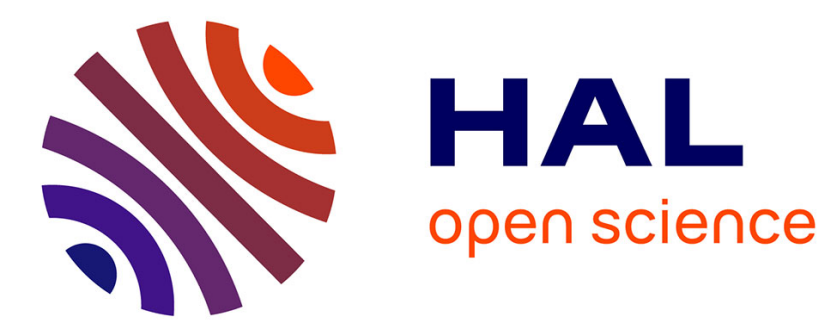

\title{
Sur la concentration de certaines fonctions additives
}

\author{
Régis de La Bretèche, Gerald Tenenbaum
}

\section{To cite this version:}

Régis de La Bretèche, Gerald Tenenbaum. Sur la concentration de certaines fonctions additives. Mathematical Proceedings of the Cambridge Philosophical Society, 2012, 152 (1), pp.179-189, 191. 10.1017/S0305004111000521 . hal-01281334v2

\section{HAL Id: hal-01281334 \\ https://hal.science/hal-01281334v2}

Submitted on 18 Jan 2017

HAL is a multi-disciplinary open access archive for the deposit and dissemination of scientific research documents, whether they are published or not. The documents may come from teaching and research institutions in France or abroad, or from public or private research centers.
L'archive ouverte pluridisciplinaire HAL, est destinée au dépôt et à la diffusion de documents scientifiques de niveau recherche, publiés ou non, émanant des établissements d'enseignement et de recherche français ou étrangers, des laboratoires publics ou privés. 


\title{
Sur la concentration de certaines fonctions additives*
}

\author{
R. de la Bretèche \& G. Tenenbaum
}

\begin{abstract}
Improving on estimates of Erdős, Halász and Ruzsa, we provide new upper and lower bounds for the concentration function of the limit law of certain additive arithmetic functions under hypotheses involving only their average behaviour on the primes. In particular we partially confirm a conjecture of Erdős and Kátai. The upper bound is derived via a reappraisal of the method of Diamond and Rhoads, resting upon the theory of functions with bounded mean oscillation.

Keywords: probabilistic number theory, Erdős-Wintner theorem, BMO functions, BerryEsseen inequality, arithmetic functions, additive functions, concentration functions, distribution of arithmetic functions.
\end{abstract}

\section{Présentation des résultats}

Le concept de fonction arithmétique additive est le pendant arithmétique de celui de somme de variables aléatoires indépendantes en théorie des probabilités. L'étude de la régularité de la répartition de tels objets est à ce titre une branche essentielle de la théorie probabiliste des nombres.

La fonction de concentration

$$
Q_{F}(h):=\sup _{z \in \mathbb{R}}\{F(z+h)-F(z)\} \quad(h>0)
$$

d'une fonction de répartition $F$ est classiquement une mesure effective de sa régularité. Dans le cas des fonctions arithmétiques, on considère usuellement le comportement asymptotique (lorsque $x \rightarrow \infty$ ) de la fonction de répartition

$$
F_{x}(z):=\frac{1}{\lfloor x\rfloor}|\{n \leqslant x: f(n) \leqslant z\}|
$$

obtenue en identifiant $f: \mathbb{N}^{*} \rightarrow \mathbb{R}$ à une variable aléatoire sur l'espace

$$
\Omega_{x}:=\{n: 1 \leqslant n \leqslant x\}
$$

muni de la probabilité uniforme.

La meilleure majoration quantitative de la fonction $Q_{F_{x}}$ est due à Ruzsa [12]. Améliorant des estimations d'Erdős [5] et Halász [9], il a montré que l'on a, uniformément lorsque $f$ décrit la classe des fonctions additives,

$$
R(x ; f):=Q_{F_{x}}(1) \ll \frac{1}{\sqrt{W_{f}(x)}}
$$

où l'on a posé

$$
W_{f}(x):=\min _{\lambda \in \mathbb{R}}\left\{\lambda^{2}+U_{f}(x, \lambda)\right\}, \quad U_{f}(x, \lambda):=\sum_{p \leqslant x} \frac{\min \left\{1,(f(p)-\lambda \log p)^{2}\right\}}{p} .
$$

2010 Mathematics Subject Classification: primary 11N60, 11N37, 11K65.

* Nous prenons en compte ici certaines corrections ultérieures à la publication de cet article. 
Ici et dans la suite, la lettre $p$ désigne exclusivement un nombre premier. Ce résultat fournit l'ordre de grandeur correct à la fois pour $f(n)=\Omega(n):=\sum_{p^{\nu} \| n} \nu$ et pour $f(n)=c \log n$. Ainsi, il découle de $(1 \cdot 1)$ que

$$
R(x ; \Omega) \ll \frac{1}{\sqrt{\log _{2} x}}, \quad R(x ; c \log ) \ll \frac{1}{c} \quad\left(1 \ll c \ll \sqrt{\log _{2} x}\right)
$$

alors que

$$
R(x ; \Omega) \sim \frac{1}{\sqrt{2 \pi \log _{2} x}}, \quad R(x ; c \log ) \sim 1-\mathrm{e}^{-1 / c} \quad(1 \ll c=o(x)) .
$$

Ici et dans la suite, nous désignons par $\log _{k}$ la $k$-ième itérée de la fonction logarithme. La première relation $(1 \cdot 2)$ résulte d'estimations classiques de type Selberg-Delange voir par exemple [13], formule (III.4.70) —; la seconde découle d'un calcul élémentaire standard.

Cependant, la majoration (1·1) est généralement moins précise lorsque $f(p)$ tend assez vite vers 0 . Ainsi, dans l'exemple historique

$$
f(n):=\log \{n / \varphi(n)\} \quad(n \geqslant 1),
$$

où $\varphi$ désigne la fonction indicatrice d'Euler, on a

$$
W_{f / \varepsilon}(x) \leqslant U_{f / \varepsilon}(x, 0) \asymp \log _{2}(1 / \varepsilon) \quad\left(0<\varepsilon \leqslant 1 / 2, x>x_{0}(\varepsilon)\right),
$$

de sorte que (1·1) fournit au mieux la majoration

$$
Q_{F_{x}}(\varepsilon) \ll \frac{1}{\sqrt{\log _{2}(1 / \varepsilon)}} \quad\left(0<\varepsilon \leqslant 1 / 2, x>x_{0}(\varepsilon)\right)
$$

alors qu'un résultat d'Erdős [6] implique, dans les mêmes conditions,

$$
Q_{F_{x}}(\varepsilon) \asymp \frac{1}{\log (1 / \varepsilon)} .
$$

Plus généralement, lorsque la fonction additive réelle $f$ vérifie les hypothèses du théorème d'Erdős-Wintner, autrement dit la convergence des trois séries

$$
\sum_{|f(p)|>1} \frac{1}{p}, \quad \sum_{|f(p)| \leqslant 1} \frac{f(p)}{p}, \quad \sum_{|f(p)| \leqslant 1} \frac{f(p)^{2}}{p},
$$

la fonction $f$ possède une fonction de répartition limite $F$, égale au produit de convolution infini $F=*_{p \in \mathbb{P}} G_{p}$, où $\mathbb{P}$ désigne l'ensemble des nombres premiers et où l'on a posé

$$
G_{p}(z):=\left(1-\frac{1}{p}\right) \sum_{f\left(p^{\nu}\right) \leqslant z} \frac{1}{p^{\nu}} \quad(p \in \mathbb{P}) .
$$

En particulier

$$
\begin{array}{ll}
1-Q_{G_{p}}(\varepsilon)=\frac{1}{p}+O\left(\frac{1}{p^{2}}\right) & (|f(p)|>\varepsilon), \\
1-Q_{G_{p}}(\varepsilon)=O\left(\frac{1}{p^{2}}\right) & (|f(p)| \leqslant \varepsilon) .
\end{array}
$$


L'inégalité de Kolmogorov-Rogozin (voir par exemple [13], th. III.2.8) implique donc

$$
Q_{F}(\varepsilon) \ll \frac{1}{\sqrt{1+\sum_{|f(p)|>\varepsilon} 1 / p}} .
$$

En majorant $W_{f / \varepsilon}(x)$ par $U_{f / \varepsilon}(x, 0)$, on peut alors vérifier facilement que la borne supérieure de (1.1) est au mieux de l'ordre de celle de (1.5).

Dans ce travail, nous nous proposons d'améliorer significativement (1.5) pour une sousclasse relativement étendue de l'ensemble des fonctions additives $f$ vérifiant

$$
\sum_{p} \frac{\min (1,|f(p)|)}{p}<\infty
$$

Il est à noter que cette condition implique la convergence des trois séries (1.4) et qu'elle lui est en fait équivalente lorsque, par exemple, $f \geqslant 0$.

Dans l'esprit des résultats de la bibliographie précédemment cités, notre objectif principal consiste ici à obtenir des bornes supérieures et inférieures pour $Q_{F}(\varepsilon)$ qui sont valables dans un cadre très général, et en particulier avec des conditions de régularité sur la suite $\{f(p)\}_{p \in \mathbb{P}}$ aussi lâches que possible.

Lorsque, à l'inverse, l'on dispose d'informations précises sur la régularité de $\{f(p)\}_{p \in \mathbb{P}}$, on peut estimer directement la fonction caractéristique de la loi de $F$, comme dans l'article d'Erdős et Kátai [7] relatif au cas $f(p):=1 /(\log p)^{c}$ avec $c \geqslant 1$, ou employer une technique élémentaire du type de celle d'Erdős dans [6] — pour le cas $0<c<2$, voir aussi Babu [1] et l'exercice 259, dû à Saffari, de [13], résolu dans [14]. L'arbitre de ce travail a obtenu par la seconde voie un résultat analogue lorsque $1<c \leqslant 2$ qui sera sans doute publié séparément.

Pour énoncer notre résultat principal, nous introduisons la notation

$$
\varepsilon_{N}=\varepsilon_{f}(N):=\sum_{\substack{p>N \\|f(p)| \leqslant 1}} \frac{|f(p)|}{p}
$$

de sorte que $\lim _{N \rightarrow \infty} \varepsilon_{f}(N)=0$ sous la condition (1.6). De plus, pour tous $w \geqslant 2$, $p \in \mathbb{P}$, nous désignons par $\left\{\varrho_{j}(p, w ; f)\right\}_{j=1}^{J}$ la suite croissante des nombres $|f(p)-f(q)|$ $(q \leqslant w, q \in \mathbb{P})$ et posons

$$
\delta_{p}(z ; f):=\varrho_{\lfloor z\rfloor}\left(p, z^{2} ; f\right) / z \quad(z \geqslant 2) .
$$

Notant $z_{j}:=2^{2^{j}}(j \geqslant 0)$, nous définissons alors

$$
\sigma_{f}^{*}(j, z):=\sum_{\substack{z_{j-1}<p \leqslant \min \left(z_{j}, z\right) \\ 0<|f(p)| \leqslant 1}} \frac{1}{p^{2} \delta_{p}\left(z_{j-1} ; f\right)} \quad(j \geqslant 1, z \geqslant 2)
$$

et

$$
\sigma_{f}(z):=\sum_{z_{j} \leqslant z^{2}} \sqrt{\sigma_{f}^{*}(j, z)} \quad(z \geqslant 2) .
$$

Nous dirons qu'une fonction arithmétique additive $f$ est admissible si elle vérifie (1·6), si

$$
f(p) \neq 0 \Rightarrow f(p) \neq f(q) \quad(p, q \in \mathbb{P}, p \neq q),
$$

et si $\mu_{f}(z):=\varepsilon_{f}(z) \sigma_{f}(z)^{2}$ est bornée. Dans ce cas, nous posons

$$
\lambda_{f}:=\limsup _{z \rightarrow \infty} \mu_{f}(z)^{1 / 3} .
$$


Il est à noter que le Théorème 1.1 infra pourrait, par la même technique, être étendu au cas où le nombre des solutions de l'équation $f(p)=f(q)$ avec $p, q \in \mathbb{P}$ est contrôlé en moyenne. Nous n'avons pas inclus une telle généralisation dans ce travail.

Nous obtenons l'énoncé suivant.

Théorème 1.1. Il existe une constante absolue $K$ telle que, pour toute fonction arithmétique additive réelle admissible $f$, on ait

$$
Q_{F}(\varepsilon) \ll \exp \left\{-\min \left(1, K / \lambda_{f}\right) \sum_{|f(p)|>\varepsilon} \frac{1-\varepsilon /|f(p)|}{p}\right\} \quad(\varepsilon \rightarrow 0+) .
$$

À l'instar du travail novateur de Diamond et Rhoads [2] que nous généralisons ici, notre méthode d'approche du Théorème 1.1 repose sur la théorie des fonctions à oscillation moyenne bornée. ${ }^{(1)}$

L'amélioration de l'inégalité de Kolmogorov-Rogozin contenue dans le Théorème 1.1 fournit génériquement l'ordre de grandeur exact, ainsi que nous le déduirons de la minoration suivante.

Théorème 1.2. Soit $f$ une fonction arithmétique additive vérifiant (1.6). Alors la fonction de répartition $F$ de la loi limite de $f$ vérifie

$$
Q_{F}(\varepsilon) \geqslant\left\{1-2 \varepsilon_{N} / \varepsilon+o(1)\right\} \prod_{p \leqslant N}\left(1-\frac{1}{p}\right) \sum_{f\left(p^{\nu}\right)=0} \frac{1}{p^{\nu}} \quad(\varepsilon \rightarrow 0),
$$

où $N=N_{\varepsilon}$ est tel que $\varepsilon_{N}=\varepsilon_{f}(N)<\frac{1}{2} \varepsilon$ lorsque $\varepsilon \rightarrow 0$.

Remarque. Dans [7], Erdős et Kátai obtiennent une minoration de l'ordre de grandeur de $Q_{F}(\varepsilon)$ en fonction de la quantité $\sum_{p>z} f(p)^{2} / p$. Ce résultat est de qualité équivalente à celui du Théorème 1.2.

Le Théorème 1.2 s'applique facilement. Le résultat suivant correspond au cas de décroissance rapide de la suite $\{f(p)\}_{p \in \mathbb{P}}$, et recouvre donc le cas classique (1·3).

Corollaire 1.3. Soient $c>0$ et $f$ une fonction arithmétique additive réelle vérifiant $|f(p)| \ll 1 / p^{c}(p \in \mathbb{P})$. Alors

$$
Q_{F}(\varepsilon) \geqslant \frac{c \mathrm{e}^{-\gamma}+o(1)}{\log 1 / \varepsilon} \quad(\varepsilon \rightarrow 0+)
$$

Lorsque

$$
f(p) \asymp 1 / p^{c}, \quad|f(p)-f(q)| \gg \min (|p-q|, p) / p^{c+1} \quad(p, q \in \mathbb{P}),
$$

on a de plus

$$
Q_{F}(\varepsilon) \ll \frac{c}{\log 1 / \varepsilon}
$$

où la constante implicite est indépendante de $c$.

Démonstration. Il est immédiat que la relation (1.6) est satisfaite. La minoration résulte donc du Théorème 1.2 en choisissant $\varepsilon_{N} \ll 1 / N^{c}$ et donc $N_{\varepsilon}=\varepsilon^{-1 / c+o(1)}$.

Sous les hypothèses supplémentaires indiquées, on vérifie sans peine à l'aide de la majoration classique de Brun-Titchmarsh (cf. par exemple [13], th. I.4.16)

$$
\pi(x+y)-\pi(x) \ll \frac{y}{\log x} \quad\left(y \geqslant x^{1 / 2}\right)
$$

que $\delta_{p}(z ; f) \gg(\log z) / p^{c}\left(z<p \leqslant z^{2}\right)$, d'où

$$
\sigma_{f}(z)^{2} \ll z^{c} /(\log z)^{2} .
$$

1. Souvent désignées par l'acronyme BMO (Bounded Mean Oscillation), voir [11]. 
Comme $\varepsilon_{f}(z) \ll 1 /\left\{z^{c} \log z\right\}$, il s'ensuit que $f$ est admissible et $\lambda_{f}=0$. La majoration résulte donc du Théorème 1.1 via un calcul standard.

Corollaire 1.4. Soient $c>1$ une constante assez grande et $f$ une fonction additive telle que $f(p) \asymp 1 /(\log p)^{c}(p \in \mathbb{P})$ et $|f(p)-f(q)| \gg|p-q| /\left\{p(\log p)^{c+1}\right\}(p, q \in \mathbb{P}, q<p)$. Alors

$$
Q_{F}(\varepsilon) \asymp \varepsilon^{1 / c} \quad(\varepsilon \rightarrow 0+) .
$$

Démonstration. Grâce à (1·11), on obtient

$$
\delta_{p}(z ; f) \gg 1 /\left\{(p+z \log p)(\log z)^{c}\right\} \quad\left(z<p \leqslant z^{4 / 3}\right),
$$

d'où, par un calcul facile,

$$
\sigma_{f}(z)^{2} \ll(\log z)^{c} .
$$

Comme $\varepsilon_{f}(z) \asymp 1 /\left\{c(\log z)^{c}\right\}$, on voit que $f$ est admissible et que $\lambda_{f} \ll c^{-1 / 3}$. En particulier $\lambda_{f}<K$ dès que $c$ est assez grand. On a

$$
\sum_{f(p)>\varepsilon} \frac{1}{p}=\frac{1}{c} \log \frac{1}{\varepsilon}+O(1), \quad \varepsilon \sum_{f(p)>\varepsilon} \frac{1}{p f(p)} \ll 1 .
$$

La majoration contenue dans (1.12) résulte donc de (1.9). La minoration résulte du choix $N_{\varepsilon} \asymp \exp \left(\varepsilon^{-1 / c}\right)$ dans le Théorème 1.2.

Remarques. (i) Dans [7], Erdős et Kátai, montrent que

$$
Q_{F}(\varepsilon) \asymp \frac{1}{\log (1 / \varepsilon)}
$$

lorsque $\varepsilon_{f}(z) \ll 1 / z^{\delta}$ et $\min _{2 \leqslant p<q \leqslant z}|f(p)-f(q)|>1 / z^{A}$ où $A$ et $\delta$ sont des constantes arbitraires. La méthode de majoration du présent travail, très générale, semble mal adaptée à ces hypothèses : elle ne fournit dans ce cas qu'une estimation du type $Q_{F}(\varepsilon) \ll\{\log (1 / \varepsilon)\}^{-c}$ où $c$ est une constante positive dépendant de $A$ et $\delta$.

(ii) Il est établi dans [7] que l'encadrement

$$
\varepsilon^{1 / c} \ll Q_{F}(\varepsilon) \ll \varepsilon^{1 / c}\left(\log _{2} 1 / \varepsilon\right)^{2} \quad\left(0<\varepsilon \leqslant \frac{1}{3}\right)
$$

est valide lorsque $f$ est la fonction fortement additive définie par $f(p):=1 /(\log p)^{c}(c>1)$. Erdős et Kátai émettent l'hypothèse que la minoration fournit l'ordre de grandeur exact pour tout $c>1$. Notre Corollaire 1.4 confirme donc cette conjecture dans un cadre général dès que $c$ est assez grand.

Grâce à un emploi canonique de l'inégalité de Berry-Esseen, le Théorème 1.1 peut être employé pour estimer la vitesse de convergence de la fonction de répartition $F_{x}$ vers celle de la loi limite $F$. Nous explicitons le résultat dans un cas particulier représentatif.

Corollaire 1.5. Soient $c>0$ et $f$ une fonction arithmétique additive réelle vérifiant $|f(p)| \ll 1 / p^{c}$ et $\sum_{p^{\nu}}\left|f\left(p^{\nu}\right)\right| / p^{\nu}<\infty$. Lorsque $x$ tend vers l'infini, on a

$$
\left\|F-F_{x}\right\|_{\infty}:=\sup _{z \in \mathbb{R}}\left|F(z)-F_{x}(z)\right| \ll_{c} Q_{F}\left(1 / T_{x}\right)+1 / \log x,
$$

où l'on a posé $T_{x}:=x^{c \log _{3} x /\left(10 \log _{2} x\right)}$.

En particulier, sous les hypothèses (1·10), nous avons

$$
\left\|F-F_{x}\right\|_{\infty} \ll_{c} \frac{\log _{2} x}{(\log x) \log _{3} x} \quad(x>16) .
$$

Le cas des fonctions $n \mapsto \log \{\varphi(n) / n\}$ et $n \mapsto \log \{\sigma(n) / n\}$ où $\sigma(n)$ désigne la somme des diviseurs de $n$ correspond à $c=1$. L'estimation (1.13) coïncide alors avec un résultat d'Elliott — [3], théorème 5.6. 


\section{Démonstrations}

\subsection{Valeurs moyennes de polynômes trigonométriques}

Le résultat suivant est une extension facile d'un résultat de Gallagher [8] fournissant une majoration de la norme quadratique d'un polynôme de Dirichlet et dont une inégalité bien connue de Montgomery et Vaughan ([10], cor.2) constitue une forme plus précise. Nous notons $\mathrm{e}(x):=\mathrm{e}^{2 \pi i x}(x \in \mathbb{R})$.

Lemme 2.1. Soient $N \in \mathbb{N}^{*},\left\{\lambda_{n}\right\}_{n=1}^{N}$ une suite finie de nombres réels distincts. Pour $1 \leqslant n \leqslant N, 1 \leqslant j<N$, on note $\left\{\varrho_{j}(n)\right\}_{n=1}^{N-1}$ la suite constituée des nombres $\left|\lambda_{n}-\lambda_{m}\right|$ $(1 \leqslant m \leqslant N, m \neq n)$ réarrangée par ordre croissant, et l'on pose $\delta_{n}(r):=\varrho_{r}(n) / r$ $(1 \leqslant r<N)$. Pour tous $\left\{a_{n}\right\}_{n=1}^{N} \in \mathbb{C}^{N}, 1 \leqslant r<N, T>0$, nous avons

$$
\int_{-T}^{T}\left|\sum_{1 \leqslant n \leqslant N} a_{n} \mathrm{e}\left(\lambda_{n} t\right)\right|^{2} \mathrm{~d} t \ll \sum_{1 \leqslant n \leqslant N}\left|a_{n}\right|^{2}\left\{r T+\frac{1}{\delta_{n}(r)}\right\},
$$

où la constante implicite est absolue.

Démonstration. Pour établir $(2 \cdot 1)$, nous observons que, posant

$$
A(x):=T \sum_{\substack{1 \leqslant n \leqslant N \\\left|x-\lambda_{n}\right| \leqslant 1 / 4 T}} a_{n}, \quad S(t):=\sum_{1 \leqslant n \leqslant N} a_{n} \mathrm{e}\left(\lambda_{n} t\right),
$$

on a

$$
\widehat{A}(t):=\int_{\mathbb{R}} A(x) \mathrm{e}(-t x) \mathrm{d} x=S(-t) \frac{\sin (\pi t / 2 T)}{\pi t / 2 T} .
$$

D'après la formule de Plancherel, nous pouvons donc écrire

$$
\int_{-T}^{T}|S(t)|^{2} \mathrm{~d} t \ll \int_{\mathbb{R}}\left|S(-t) \frac{\sin (\pi t / 2 T)}{\pi t / 2 T}\right|^{2} \mathrm{~d} t=\int_{\mathbb{R}}|A(x)|^{2} \mathrm{~d} x .
$$

Posons alors $N_{k}:=\sum_{\left|2 T \lambda_{n}-k\right| \leqslant 1}\left|a_{n}\right|(k \in \mathbb{Z})$ et observons que $|A(x)| \leqslant T N_{k}$ lorsque $|x-k / 2 T| \leqslant 1 / 4 T$. Il suit

$$
\int_{\mathbb{R}}|A(x)|^{2} \mathrm{~d} x \ll T \sum_{k \in \mathbb{Z}} N_{k}^{2}
$$

Notons $\left\{\lambda_{j}^{*}: 1 \leqslant j \leqslant J_{k}\right\}$ la suite des $\lambda_{n}$ comptés dans $N_{k}$, réordonnée par ordre croissant, et posons $b_{j}:=\left|a_{n}\right|$ si $\lambda_{j}^{*}=\lambda_{n}, \delta_{j}^{*}:=T\left(\lambda_{j+r}^{*}-\lambda_{j}^{*}\right)\left(1 \leqslant j \leqslant J_{k}-r\right)$. Si $J_{k} \leqslant r$, nous avons clairement $N_{k}^{2} \leqslant r \sum_{\left|2 T \lambda_{n}-k\right| \leqslant 1}\left|a_{n}\right|^{2}$. Dans le cas contraire, nous pouvons écrire, en vertu de l'inégalité de Cauchy-Schwarz,

$$
\begin{aligned}
N_{k}^{2} & \leqslant T \sum_{1 \leqslant j \leqslant J_{k}-r}\left(\lambda_{j+r}^{*}-\lambda_{j}^{*}\right) \sum_{1 \leqslant j<J_{k}} \frac{b_{j}^{2}}{\delta_{j}^{*}}+r \sum_{J_{k}-r<j \leqslant J_{k}} b_{j}^{2} \\
& \leqslant r \sum_{1 \leqslant j \leqslant J_{k}-r} \frac{b_{j}^{2}}{\delta_{j}^{*}}+r \sum_{J_{k}-r<j \leqslant J_{k}} b_{j}^{2} \leqslant \sum_{\left|2 T \lambda_{n}-k\right| \leqslant 1}\left|a_{n}\right|^{2}\left(r+\frac{1}{T \delta_{n}(r)}\right) .
\end{aligned}
$$

En sommant sur $k$, nous obtenons bien l'inégalité annoncée.

\subsection{Un résultat d'oscillation moyenne bornée}

Étant donnés $\alpha$ et $\beta \in \mathbb{R}, 2 \leqslant \alpha<\beta$, nous posons

$$
S_{\alpha, \beta}(t):=S(t)=\sum_{\substack{\alpha<p \leqslant \beta \\ 0<|f(p)| \leqslant 1}} \frac{\cos (t f(p))}{p} .
$$


Le lemme suivant montre que, sous des conditions peu restrictives concernant $f$, la fonction $S$ est à oscillation moyenne bornée. La démonstration simplifie et généralise celle de Diamond et Rhoads [2].

Lemme 2.2. Pour toute fonction arithmétique additive réelle admissible $f$, on a

$$
\sup _{a<b} \frac{1}{b-a} \int_{a}^{b}\left|S(t)-E_{I}(S)\right| \mathrm{d} t \ll \frac{1}{\sqrt{\log \alpha}}+\sup _{\alpha \leqslant z \leqslant \beta} \mu_{f}(z)^{1 / 3}
$$

où $E_{I}(S)$ désigne la valeur moyenne de $S$ sur $I:=[a, b]$.

Démonstration. Nous pouvons supposer sans perte de généralité que $\alpha$ et $\beta$ sont entiers.

Plaçons-nous d'abord dans la circonstance où $b-a \leqslant\left\{\sigma_{f}(\alpha) / \varepsilon_{f}(\alpha)\right\}^{2 / 3}$. Il existe $v \in I$ tel que $E_{I}(S)=S(v)$; la quantité à majorer est donc trivialement

$$
\leqslant \frac{1}{b-a} \int_{a}^{b} \sum_{\substack{\alpha<p \leqslant \beta \\|f(p)| \leqslant 1}} \frac{|f(p)(t-v)|}{p} \mathrm{~d} t \leqslant \frac{1}{2}(b-a) \varepsilon_{f}(\alpha) \leqslant \frac{1}{2} \mu_{f}(\alpha)^{1 / 3},
$$

d'où $(2 \cdot 2)$.

Considérons ensuite le cas $b-a>\left\{\sigma_{f}(\beta) / \varepsilon_{f}(\beta)\right\}^{2 / 3}$. Nous scindons la somme $S(t)$ aux points $z_{j}$, et appliquons à chacune des sous-sommes, disons $S_{j}$, la majoration

$$
\left(\frac{1}{b-a} \int_{a}^{b}\left|S_{j}(t)-E_{I}\left(S_{j}\right)\right| \mathrm{d} t\right)^{2} \leqslant \frac{1}{b-a} \int_{a}^{b}\left|S_{j}(t)-Y\right|^{2} \mathrm{~d} t \quad(Y \in \mathbb{R})
$$

avec $Y:=0$.

Employant alors le Lemme 2.1, nous obtenons

$$
\begin{aligned}
\frac{1}{b-a} \int_{a}^{b}\left|S(t)-E_{I}(S)\right| \mathrm{d} t & \\
& \ll \sum_{\alpha^{1 / 2}<z_{j} \leqslant \beta^{2}}\left\{\sum_{\substack{z_{j-1<p \leqslant z_{j}} \\
0<|f(p)| \leqslant 1}} \frac{1}{p^{2}}\left(z_{j-1}+\frac{1}{(b-a) \delta_{p}\left(z_{j-1} ; f\right)}\right)\right\}^{1 / 2} \\
& \ll \frac{1}{\sqrt{\log \alpha}}+\frac{\sigma_{f}(\beta)}{\sqrt{b-a}} \ll \frac{1}{\sqrt{\log \alpha}}+\mu_{f}(\beta)^{1 / 3} .
\end{aligned}
$$

Cela implique bien $(2 \cdot 2)$.

Il reste à traiter le cas où $\left\{\sigma_{f}(\alpha) / \varepsilon_{f}(\alpha)\right\}^{2 / 3}<b-a \leqslant\left\{\sigma_{f}(\beta) / \varepsilon_{f}(\beta)\right\}^{2 / 3}$. Il existe alors un entier $\gamma \in[\alpha, \beta[$ tel que

$$
\left\{\sigma_{f}(\gamma) / \varepsilon_{f}(\gamma)\right\}^{2 / 3} \leqslant b-a<\left\{\sigma_{f}(\gamma+1) / \varepsilon_{f}(\gamma+1\}^{2 / 3}\right.
$$

Notant

$$
J(\alpha, \beta):=\frac{1}{b-a} \int_{a}^{b}\left|S_{\alpha, \beta}(t)-E_{I}\left(S_{\alpha, \beta}\right)\right| \mathrm{d} t,
$$

nous pouvons écrire

$$
\begin{aligned}
J(\alpha, \beta) & \leqslant J(\alpha, \gamma)+J(\gamma, \gamma+1)+J(\gamma+1, \beta) \\
& \ll \frac{1}{\sqrt{\log \alpha}}+\mu_{f}(\gamma)^{1 / 3}+\frac{1}{\gamma+1}+\mu_{f}(\gamma+1)^{1 / 3} .
\end{aligned}
$$

Cela complète la démonstration puisque $\gamma \geqslant \alpha$. 


\subsection{Preuve du Théorème 1.1}

Soit

$$
\widehat{F}(\tau)=\prod_{p}\left(1-\frac{1}{p}\right) \sum_{\nu \geqslant 0} \frac{\mathrm{e}^{i \tau f\left(p^{\nu}\right)}}{p^{\nu}} \quad(\tau \in \mathbb{R})
$$

la fonction caractéristique de $F$. Pour tous $\alpha, \beta$ tels que $2 \leqslant \alpha<\beta$, nous pouvons écrire

$$
|\widehat{F}(\tau)| \ll \prod_{\substack{\alpha<p \leqslant \beta \\ 0<|f(p)| \leqslant 1}}\left(1-\frac{1}{p}\right)\left|1+\frac{\mathrm{e}^{i \tau f(p)}}{p}\right| \ll \mathrm{e}^{S_{\alpha, \beta}(\tau)} \prod_{\substack{\alpha<p \leqslant \beta \\ 0<|f(p)| \leqslant 1}}\left(1-\frac{1}{p}\right) \quad(\tau \in \mathbb{R}) .
$$

De plus, nous avons classiquement (voir par exemple le lemme III.2.9 de [13])

$$
Q_{F}(\varepsilon) \ll \varepsilon \int_{-1 / \varepsilon}^{1 / \varepsilon}|\widehat{F}(\tau)| \mathrm{d} \tau
$$

Bien entendu, comme $|\widehat{F}(\tau)| \leqslant 1$, nous pouvons également élever l'intégrande à une puissance positive quelconque n'excédant pas l'unité sans altérer la validité de cette estimation.

Comme dans [2], nous faisons appel au résultat de [11] selon lequel il existe une constante absolue $b>0$ telle que, si $B$ désigne un majorant quelconque du membre de gauche de $(2 \cdot 2)$, on ait, uniformément pour $\varepsilon>0$ et pour le choix $I:=[-1 / \varepsilon, 1 / \varepsilon]$,

$$
\varepsilon \int_{-1 / \varepsilon}^{1 / \varepsilon} \mathrm{e}^{b S_{\alpha, \beta}(\tau) / B} \mathrm{~d} \tau \ll \mathrm{e}^{b\left|E_{I}\left(S_{\alpha, \beta}\right)\right| / B}
$$

Soit $M$ la constante implicite dans $(2 \cdot 2)$. Nous choisissons $\alpha$ suffisamment grand pour que le membre de gauche de $(2 \cdot 2)$ n'excède pas $B:=\frac{1}{2} b+M \lambda_{f}$ et observons que

$$
\begin{aligned}
\left|E_{I}\left(S_{\alpha, \beta}\right)\right|:=\frac{1}{2} \varepsilon\left|\int_{-1 / \varepsilon}^{1 / \varepsilon} S_{\alpha, \beta}(\tau) \mathrm{d} \tau\right| & \leqslant \varepsilon \sum_{\substack{\alpha<p \leqslant \beta \\
0<|f(p)| \leqslant 1}} \frac{|\sin (f(p) / \varepsilon)|}{p|f(p)|} \\
& \leqslant \sum_{\substack{\alpha<p \leqslant \beta \\
0<|f(p)| \leqslant 1}} \frac{\min \{1, \varepsilon /|f(p)|\}}{p} .
\end{aligned}
$$

Il suit, pour $c:=\min (1, b / B)$,

$$
Q_{F}(\varepsilon) \ll \varepsilon \int_{-1 / \varepsilon}^{1 / \varepsilon}|\widehat{F}(\tau)|^{c} \mathrm{~d} \tau \ll \prod_{\substack{\alpha<p \leqslant \beta \\ 0<|f(p)| \leqslant 1}}\left(1-\frac{1}{p}\right)^{c} \exp \left\{c \sum_{\substack{\alpha<p \leqslant \beta \\ 0<|f(p)| \leqslant 1}} \frac{\min \{1, \varepsilon /|f(p)|\}}{p}\right\} .
$$

Cela implique le résultat annoncé en faisant tendre $\beta$ vers l'infini et en observant que $c \geqslant \min \left(1, K / \lambda_{f}\right)$ pour le choix $K:=b / 2 M$.

2.4. Preuve du Théorème 1.2

Soit

$$
\mathcal{A}_{N}:=\left\{\begin{aligned}
& (\forall p \leqslant N) p^{\nu} \| n \Rightarrow f\left(p^{\nu}\right)=0, \\
n \in \mathbb{N}^{*}: & (\forall p>N) p \mid n \Rightarrow p^{2} \nmid n, \\
& (\forall p>N)|f(p)|>1 \Rightarrow p \nmid n
\end{aligned}\right\} .
$$


Posons

$$
C_{N}:=\prod_{p>N}\left(1-\frac{1}{p^{2}}\right) \prod_{\substack{p>N \\|f(p)|>1}}\left(\frac{1-1 / p}{1-1 / p^{2}}\right) \prod_{p \leqslant N}\left(1-\frac{1}{p}\right) \sum_{f\left(p^{\nu}\right)=0} \frac{1}{p^{\nu}} .
$$

Lorsque $x$ tend vers l'infini, nous avons

$$
\sum_{\substack{n \leqslant x \\ n \in \mathcal{A}_{N}}} 1 \sim C_{N} x, \quad \sum_{\substack{n \leqslant x \\ n \in \mathcal{A}_{N}}}|f(n)| \leqslant \varepsilon_{N} C_{N} x+o(x) .
$$

Il s'ensuit que

$$
F\left(\frac{1}{2} \varepsilon\right)-F\left(-\frac{1}{2} \varepsilon\right) \geqslant \operatorname{dens}\left\{n \in \mathcal{A}_{N}:|f(n)| \leqslant \frac{1}{2} \varepsilon\right\} \geqslant\left\{1-2 \varepsilon_{N} / \varepsilon\right\} C_{N} .
$$

On obtient le résultat indiqué en spécialisant $N=N_{\varepsilon}$ puisque $N_{\varepsilon} \rightarrow \infty$ lorsque $\varepsilon \rightarrow 0$.

\subsection{Preuve du Corollaire 1.5}

Désignons par $\widehat{F}$ et $\widehat{F}_{x}$ les fonctions caractéristiques respectives de $F$ et $F_{x}$. L'inégalité de Berry-Esseen — voir par exemple Elliott [4], lemme 1.47 — s'écrit

$$
\left\|F-F_{x}\right\|_{\infty} \ll Q_{F}\left(\frac{1}{T}\right)+\int_{-T}^{T}\left|\widehat{F}(\tau)-\widehat{F}_{x}(\tau)\right| \frac{\mathrm{d} \tau}{|\tau|} \quad(T>0) .
$$

La Corollaire 1.5 est donc une conséquence immédiate du lemme suivant, dont la démonstration est calquée sur celle du cas $f(n)=\log (n / \varphi(n))$ traité par Elliott [3].

Lemme 2.3. Dans les hypothèses du Corollaire 1.5, nous avons

$$
\widehat{F}_{x}(\tau)=1+O(\tau) \quad(\tau \in \mathbb{R}), \quad \widehat{F}(\tau)-\widehat{F}_{x}(\tau) \ll_{c} 1 /(\log x)^{2} \quad\left(|\tau| \leqslant T_{x}\right) .
$$

Démonstration. La première estimation découle immédiatement de la majoration

$$
\widehat{F}_{x}(\tau)-1 \ll \frac{\tau}{x} \sum_{n \leqslant x}|f(n)| \ll \tau \sum_{p^{\nu} \leqslant x} \frac{\left|f\left(p^{\nu}\right)\right|}{p^{\nu}} .
$$

Pour démontrer la seconde, nous introduisons la fonction multiplicative $h:=\mu * \mathrm{e}^{i \tau f}$, de sorte que $h\left(p^{\nu}\right)=\mathrm{e}^{i \tau f\left(p^{\nu}\right)}-\mathrm{e}^{i \tau f\left(p^{\nu-1}\right)}(p \in \mathbb{P}, \nu \geqslant 1)$ et

$$
\widehat{F}(\tau)-\widehat{F}_{x}(\tau)=\sum_{d \geqslant 1} h(d)\left(\frac{1}{d}-\frac{1}{x}\left[\frac{x}{d}\right]\right)=\frac{1}{x} \sum_{d \geqslant 1} h(d)\left\langle\frac{x}{d}\right\rangle,
$$

où $\langle t\rangle$ désigne la partie fractionnaire du nombre réel $t$. Pour tout $\vartheta \in] 0,1[$, il vient

$$
\widehat{F}(\tau)-\widehat{F}_{x}(\tau) \ll \sum_{d \geqslant 1} x^{-\vartheta} \frac{|h(d)|}{d^{1-\vartheta}} \ll x^{-\vartheta} \exp \left(\sum_{p} \frac{|h(p)|}{p^{1-\vartheta}}\right) .
$$

Choisissons $\vartheta:=5\left(\log _{2} x\right) / \log x$ et posons $P_{x}:=T_{x}^{1 / c}$. Lorsque $|\tau| \leqslant T_{x}$, nous pouvons écrire

$$
\sum_{p>P_{x}} \frac{|h(p)|}{p^{1-\vartheta}} \ll \tau \sum_{p>P_{x}} \frac{|f(p)|}{p^{1-\vartheta}} \ll T_{x} \sum_{p>P_{x}} \frac{1}{p^{1+c-\vartheta}} \ll 1 .
$$

De plus,

$$
\sum_{p \leqslant P_{x}} \frac{|h(p)|}{p^{1-\vartheta}} \leqslant 2 \sum_{p \leqslant P_{x}} \frac{1}{p^{1-\vartheta}} \leqslant 2 \log _{2} P_{x}+O\left(\vartheta\left(\log P_{x}\right) P_{x}^{\vartheta}+1\right) \leqslant\{2+o(1)\} \log _{2} x .
$$

En reportant dans $(2 \cdot 3)$, il suit

$$
\widehat{F}(\tau)-\widehat{F}_{x}(\tau) \ll x^{-\vartheta}(\log x)^{2+o(1)} \ll 1 /(\log x)^{2} .
$$

Remerciements. Les auteurs expriment leurs chaleureux remerciements à l'arbitre pour sa relecture attentive et ses conseils judicieux. 


\section{Bibliographie}

[1] G. J. Babu, Absolutely continuous distribution functions of additive functions $f(p)=(\log p)^{-a}$, $a>0$, Acta Arith. 26 n 4 (1974/75), 401-403.

[2] H.G. Diamond \& D. Rhoads, The modulus of continuity of the distribution function of $\varphi(n) / n$, Topics in classical number theory, Vol. I, II (Budapest, 1981), 335-353, Colloq. Math. Soc. János Bolyai, 34, North-Holland, Amsterdam, 1984.

[3] P.D.T.A. Elliott, Probabilistic number theory, I : mean value theorems, Grundlehren der Math. Wiss. 239, Springer-Verlag, New York, Berlin, Heidelberg 1979.

[4] P.D.T.A. Elliott. Probabilistic number theory, II : central limit theorems, Grundlehren der Math. Wiss. 240, Springer, New York, 1980.

[5] P. Erdős, On the distribution function of additive functions, Ann. of Math. 47 (1946), 1-20.

[6] P. Erdős, On the distribution of numbers of the form $\sigma(n) / n$ and on some related questions, Pacific J. Math. 52 (1974), 59-65.

[7] P. Erdős \& I. Kátai, On the concentration of distribution of additive functions, Acta Sci. Math. (Szeged) $41 \mathrm{n}^{\mathrm{os}} 3-4$ (1979), 295-305.

[8] P.X. Gallagher, A large sieve density estimate near $\sigma=1$, Invent. Math. 11 (1970), 329-339.

[9] G. Halász, On the distribution function of additive arithmetical functions, Acta Arith. 27 (1975), 143-152.

[10] H.L. Montgomery \& R.C. Vaughan, Hilbert's inequality, J. London Math. Soc. (2) 8 (1974), 73-82.

[11] F. John \& L. Nirenberg, On functions of bounded mean oscillation, Comm. Pure Appl. Math., 14 (1961), 415-426.

[12] I.Z. Ruzsa, On the concentration of additive functions, Acta Math. Acad. Sci. Hungar. 36 (1980), no. 3-4, 215-232.

[13] G. Tenenbaum, Introduction à la théorie analytique et probabiliste des nombres, troisième édition, coll. Échelles, Belin, 2008.

[14] G. Tenenbaum, avec la collaboration de J. Wu, Exercices corrigés de théorie analytique et probabiliste des nombres, Cours spécialisés, no 2, Société Mathématique de France (1996), xiv + $251 \mathrm{pp}$.

Régis de la Bretèche

Institut de Mathématiques de Jussieu

Université Paris Diderot-Paris 7

UFR de Mathématiques, Case 7012

Bâtiment Chevaleret

75205 Paris Cedex 13

France

breteche@math.jussieu.fr
Gérald Tenenbaum

Institut Élie Cartan

Université de Nancy 1

BP 239

54506 Vandœuvre Cedex

France

gerald.tenenbaum@iecn.u-nancy.fr 